\title{
INEQUALITIES FOR DUAL QUERMASSINTEGRALS \\ OF THE $p$-CROSS-SECTION BODIES
}

\author{
WEIDONG WANG AND LI YAN
}

Abstract. Gardner and Giannopoulos defined the $p$-cross-section body $C_{p} K(p>-1)$ of convex body $K$ in Euclidean space $\mathbb{E}^{n}$. In this paper, we obtain inequalities for dual quermassintegrals of the $p$-cross-section body $C_{p} K$. Further, two monotonic inequalities concerning the $C_{p} K$ are given.

Mathematics subject classification (2010): 52A40, 52A20, 52A39.

Keywords and phrases: p-cross-section body, dual quermassintegrals, intersection body, monotonic.

\section{REFERENCES}

[1] U. BREHM, Convex bodies with nonconvex cross-section bodies, Mathematika 46, 1 (1999), $127-129$.

[2] W. J. FIREY, p-means of convex bodies, Math Scand. 10 (1962), 17-24.

[3] R. J. Gardner, Geometric Tomography, Second ed., Cambridge Univ. Press, Cambridge, 2006.

[4] R. J. Gardner And A. A. Giannopoulos, p-cross-section bodies, Indiana U. Math. J. 48 (1999), 593-613.

[5] R. J. Gardner And G. Y. Zhang, Affine inequalities and radial mean bodies, Amer. J. Math. 120 (1998), 505-528.

[6] E. Grinberg And G. Y. Zhang, Convolutions, transforms, and convex bodies, Proc. London Math. Soc. 78 (1999), 77-115.

[7] C. Haberl, $L_{p}$ intersection bodies, Adv. Math. 217 (2008), 2599-2624.

[8] C. HABERL AND M. LudWIG, A characterization of $L_{p}$ intersection bodies, International Mathematics Research Notices (Int. Math. Res. Not.), 2006, Art ID 10548, 29 pages.

[9] C. HABERL AND F. SChUSTER, General $L_{p}$ affine isoperimetric inequalities, J. Differential Geom. 83 (2009), 1-26.

[10] G. H. Hardy, J. E. Littlewood and G. Pólya, Inequalities, Cambridge University Press, Cambridge, 1959.

[11] E. Lutwak, Dual mixed volumes, Pacific J. Math. 58 (1975), 531-538.

[12] E. LuTwaK, The Brunn-Minkowski-Firey theory I: mixed volumes and the minkowski problem, J. Differential Geom. 38 (1993), 131-150.

[13] E. LutwaK, The Brunn-Minkowski-Firey theory II: affine and geominimal surface areas, Adv. Math. 118 (1996), 244-294.

[14] E. LUTwAK, D. YANG AND G. Y. ZHANG, $L_{p}$ affine isoperimetric inequalities, J. Differential Geom. 56 (2000), 111-132.

[15] E. Lutwak, D. Yang And G. Y. Zhang, Sharp affine $L_{p}$ Sobolev inequalities, J. Differential Geom. 52 (2002), 17-38.

[16] E. Lutwak, D. Yang And G. Y. Zhang, On the $L_{p}$-Minkowski problem, Trans. Amer. Math. Soc. 356 (2004), 4359-4370.

[17] E. Lutwak, D. Yang And G. Y. Zhang, $L_{p}$ John ellipsoids, Proc. London Math. Soc. 90 (2005), 497-520.

[18] E. Lutwak, D. YAng AND G. Y. ZHAng, Volume inequalities for subspace of $L_{p}$, J. Differential Geom. 68 (2004), 159-184.

[19] E. LutwaK AND G. Y. Zhang, Blaschke-Santalo inequalities, J. Differential Geom. 47 (1997), $1-16$. 
[20] T. Y. MA AND W. D. WANG, On the analog of Shephard problem for the $L_{p}$-projection body, Math. Ineq. Appl. 14 (2011), 181-192.

[21] M. MEYER, Maximal hyperplane sections of convex bodies, Mathematika 46, 1 (1999), 131-136.

[22] R. SchneIder, Convex Bodies: The Brunn-Minkowski Theory, Cambridge Univ. Press, Cambridge, Second Expanded Edition, 2014.

[23] W. WANg And B. W. He, $L_{p}$-dual affine surface area, J. Math. Anal. Appl. 348 (2008), 746-751.

[24] W. WANG AND B. W. HE, Inequalities for $L_{p}$-dual affine surface area, Math. Ineq. Appl. 13 (2010), 319-327.

[25] W. D. Wang And G. S. Leng, $L_{p}$-dual mixed quermassintegrals, Indian J. Pure Appl. Math. 36 (2005), 177-188.

[26] W. D. WAng, F. H. LU AND G. S. LENG, A type of monotonicity on the $L_{p}$ centroid body and $L_{p}$ projection body, Math. Ineq. Appl. 8 (2005), 735-742.

[27] W. D. WANG AND C. QI, $L_{p}$-dual geominimal surface area, J. Inequal. Appl. 2011, 6 (2011), 10 pages.

[28] W. D. WANG AND Y. P. Zhou, Inequalities for the p-cross-section bodies, Math. Ineq. Appl. 17, 3 (2014), 1005-1013.

[29] E. Werner And D. Ye, New $L_{p}$-affine isoperimetric inequalities, Adv. Math. 218 (2008), 762-780.

[30] J. Yuan And C. W. Sum, $L_{p}$-intersection bodies, J. Math. Anal. Appl. 339 (2008), 1431-1439. 